\title{
Bariatric Surgery in Morbidly Obese Adolescents: a Systematic Review and Meta-analysis
}

\author{
Givan F. Paulus • Loes E. G. de Vaan • \\ Froukje J. Verdam • Nicole D. Bouvy • \\ Ton A. W. Ambergen • L. W. Ernest van Heurn
}

Published online: 20 February 2015

(C) The Author(s) 2015. This article is published with open access at Springerlink.com

\begin{abstract}
Pubmed, Embase, and Cochrane were systematically reviewed for available evidence on bariatric surgery in adolescents. Thirty-seven included studies evaluated the effect of laparoscopic adjustable gastric banding (LAGB), Roux-enY gastric bypass (RYGB), or laparoscopic sleeve gastrectomy (LSG) in patients $\leq 18$ years old. Fifteen of 37 studies were prospective, including one RCT. Mean body mass index (BMI) loss after LAGB was $11.6 \mathrm{~kg} / \mathrm{m}^{2}$ (95\% CI 9.8-13.4), versus $16.6 \mathrm{~kg} / \mathrm{m}^{2}$ (95\% CI 13.4-19.8) after RYGB and $14.1 \mathrm{~kg} / \mathrm{m}^{2}$ (95\% CI 10.8-17.5) after LSG. Two unrelated deaths were reported after 495 RYGB procedures. All three bariatric procedures result in substantial weight loss and improvement of comorbidity with an acceptable complication rate, indicating that surgical intervention is applicable in appropriately selected morbidly obese adolescents.
\end{abstract}

Keywords Adolescents $\cdot$ RYGB $\cdot$ LAGB $\cdot$ LSG $\cdot$ Bariatric surgery $\cdot$ Meta-analysis $\cdot$ Review $\cdot$ Weight loss $\cdot$ Quality of life

G. F. Paulus $(\bowtie) \cdot$ L. E. G. de Vaan · F. J. Verdam • N. D. Bouvy L. W. E. van Heurn

Department of Surgery, Maastricht University Medical Center, and Nutrition and Toxicology Research Institute (NUTRIM), P.

Debyelaan 25, 6229 HX Maastricht, The Netherlands

e-mail: research@givan.nl

T. A. W. Ambergen

Department of Methodology and Statistics, Maastricht University,

Maastricht, The Netherlands

F. J. Verdam

Department of Otolaryngology, University Medical Centre Utrecht, Utrecht, The Netherlands

\section{Introduction}

Obesity is an emerging pandemic phenomenon [1]. Over the past three decades, the prevalence of adult obesity in the USA has doubled, while that of adolescent obesity has tripled [2]. Current estimates classify $33.6 \%$ of adolescents living in the USA as overweight, $18.4 \%$ as obese, and $13.0 \%$ as being extremely obese, defined as body mass index (BMI) $\geq 85$ th, 95th, and 97th percentile, respectively [3]. Individual, social, environmental, and economic factors contribute to the development and persistence of morbid obesity.

Adolescent obesity is associated with preventable chronic health conditions like type two diabetes mellitus (T2DM), hypertension, obstructive sleep apnea syndrome (OSAS), dyslipidemia, nonalcoholic steatohepatitis, polycystic ovary syndrome, and various musculoskeletal diseases [4, 5]. Obese adolescents are likely to suffer from psychological morbidity, loss of self-esteem, and social exclusion which has the potential to scar them for life [6]. The risk of dying from any obesity-related cause increases by $6-7 \%$ for every 2 years lived with obesity [7]. These findings urge us to find ways to treat obesity early in life.

Presently, adolescent obesity is mostly managed by combined lifestyle interventions focusing on behavioral and dietary modifications. These treatments are typically initiated and evaluated by a multidisciplinary team including a pediatrician, dietician, psychologist, and a physiotherapist. While often effective in short term, long-term effects are relatively disappointing. A recent Cochrane review shows a maximum of $1.7 \mathrm{~kg} / \mathrm{m}^{2}$ BMI loss after 12 months of lifestyle intervention [8].

In adults, bariatric surgery is extremely effective compared to conservative treatment, resulting in adequate long-term weight loss and reduction of mortality [9]. The last decades, various bariatric procedures have been performed in adolescents, including laparoscopic adjustable gastric banding 
(LAGB), Roux-en-Y gastric bypass (RYGB), vertical banded gastroplasty, biliopancreatic diversion, and more recently laparoscopic sleeve gastrectomy (LSG). Potential adverse effects on growth and development in prepubertal patients who have not reached full maturity raise concerns. However, bariatric surgery relatively early in life intervenes before comorbidities become irreversible and reduces the risk of surgical complications.

Currently, the guidelines from the International Pediatric Endosurgery Group (IPEG) state that adolescents with a BMI $>40 \mathrm{~kg} / \mathrm{m}^{2}$ or a BMI $>35 \mathrm{~kg} / \mathrm{m}^{2}$ combined with severe comorbidities should be considered for surgical intervention, if they have (nearly) attained adult stature [10]. These guidelines are largely based upon a systematic review and metaanalysis by Treadwell et al. [11], reviewing studies up to December 2007. The last few years, indication criteria for bariatric surgery have expanded, and surgical techniques have improved. However, the outcome and best techniques to treat morbidly obese adolescents remain relatively unknown.

In this review, we evaluate and compare the efficacy, safety, and (psychosocial) health benefits of various bariatric surgical techniques as a treatment for morbid obesity in adolescents. Our data are obtained with help of supplemental data from several authors and strengthened by inclusion of the most recent high-quality studies.

\section{Methods}

Protocol and Registration

This review was conducted according to the PRISMA [12] and MOOSE [13] statements.
Eligibility Criteria

Prospective clinical trials and observational studies on LAGB, RYGB, and LSG were included with the following inclusion criteria: $\geq 10$ patients, mean follow-up $\geq 12$ months, age $\leq 18$ years at time of operation (and less than $20 \%>18$ years), majority of procedures $<25$ years ago, and English full-text available. Metaanalysis of BMI loss was done when BMI loss was either reported or could be calculated.

Search

Pubmed, Embase, and Cochrane databases were searched on the 20 January 2014 with relevant search terms and Medical Subject Headings (MeSH) on LAGB, RYGB, and LSG in children and adolescents. Full electronic Pubmed search is presented in Fig. 1.

\section{Study Selection}

After electronically removing duplicates using EndNote X6.0.1 (Thomson Reuters), all remaining duplicate entries and aberrant records were manually removed. Two independent researchers (GP and LdV) screened the remaining abstracts and/or full-text version and collected the eligible citations. Clinical data and study properties were added to the citations by reviewing all full-text articles. Reviewing inclusion period, surgical center, authors, and population characteristics identified publications with data overlap; in which case, articles presenting the most complete and/or recent data were included.

Fig. 1 Search terms: full Pubmed search

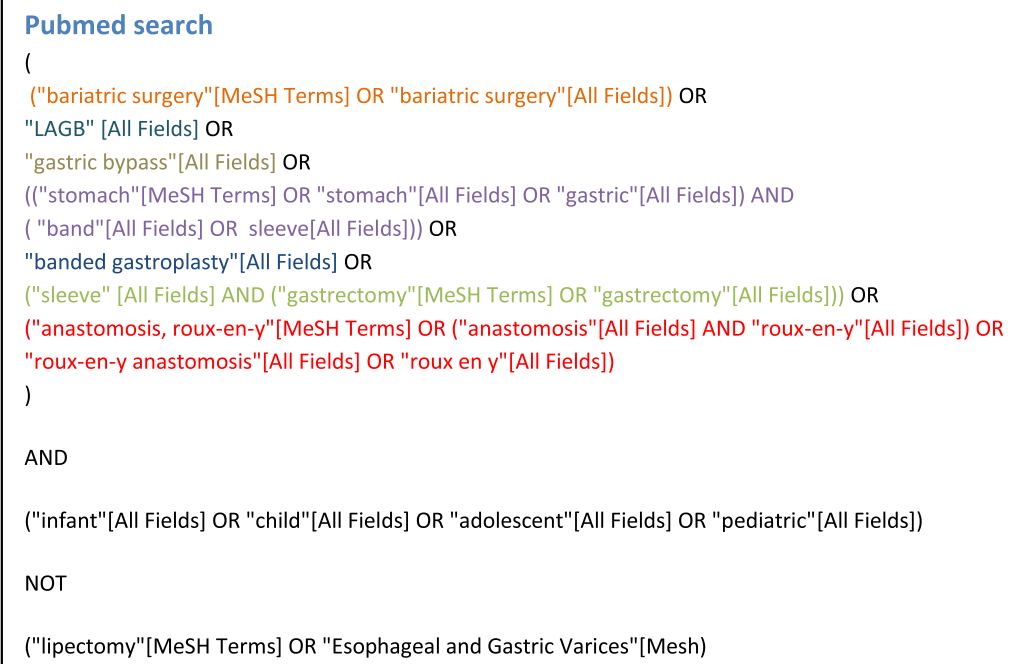




\section{Data Collection Process}

Data relevant for our systematic review and meta-analysis were collected in a datasheet and completed with data from referenced articles or previous publications or by contacting the corresponding author.

\section{Data Items}

BMI before and after the procedure or BMI loss with reported variance, complications, and change in comorbidity was extracted from each article. When individual patient data were available, mean BMI and variance were calculated for those patients younger than 19 years. Mean BMI at follow-up was only used to calculate BMI loss if more than $50 \%$ of the baseline population had reached that moment.

\section{Risk of Bias in Individual Studies}

Study characteristics that influence risk of bias (e.g. prospective/retrospective) were assessed and collected in a table. Additionally, two independent reviewers carefully assessed details on the in- and exclusion process, preoperative lifestyle treatment, postoperative lifestyle support and loss to followup.

\section{Summary Measures}

Mean BMI loss was used for meta-analysis. Corresponding authors were contacted if variance of BMI loss was not reported. Complications and comorbidity resolution were summarized if follow-up was at least 6 months. Minor complications, reported in less than three studies, were omitted from the results.

\section{Synthesis of Results}

Summary effect measure of BMI loss and forest plots were produced with $95 \%$ CI for each surgical method using STATA (StataCorp. 2013. Stata Statistical Software: Release 13. College Station, TX, USA). Differences between operative techniques were tested in a random effect model. For missing variances, the square root of the average sample-sizeweighted variance from all available variances was used. Data on complications or comorbidities were summarized when they were specifically mentioned. Results from large multicenter database studies were not summarized, while for shortterm studies ( $<6$-month follow-up), only perioperative results were summarized.

\section{Risk of Bias Across Studies}

A funnel plot for standard error of BMI loss against BMI loss was used to assess publication bias for each technique. The straight lines indicate the region within which $95 \%$ of points should lie in the absence of both heterogeneity and publication bias (Fig. 4).

\section{Additional Analyses}

A meta-regression analysis was performed to assess if BMI loss was affected by follow-up duration after the first 12 months or by different surgical gastric banding techniques (perigastric vs. pars flaccida). Authors were contacted when technical details were not provided. Additionally, differences in baseline BMI of different surgical procedures were tested in a random effect model.

\section{Results}

\section{Study Selection}

The search in Pubmed, Embase, and Cochrane provided a total of 4575 citations. After removing duplicates and screening abstracts, 4468 records were excluded and 107 remained for full-text analysis. Seventy full-text articles did not meet the inclusion criteria. Therefore, a total of 37 articles were included, including one article reporting on both LAGB and LSG. Eleven of 18 LAGB studies, 6 of 13 RYGB studies, and 5 of 7 LSG studies were eligible for meta-analysis of BMI loss (Table 1, Fig. 2). No additional studies were identified through cross-referencing.

\section{Risk of Bias Within Studies}

The study design (randomized control trial (RCT), prospective, and retrospective) and study characteristics are presented in Table 1. Potential introducers of bias, other than design, are reported in Table 2. Of 18 LAGB studies, seven were prospective, including the only RCT in this review. Five of 13 RYGB studies were prospective and three of seven LSG studies.

\section{Results of Individual Studies}

In 15 of the 22 included datasets, SD of BMI loss was not reported or available. Nine of the contacted research groups were willing to supply data on BMI loss with SD at one or more follow-up moments to complete the dataset. Finally, 14 SDs were available and 8 were derived as stated in the methods. 


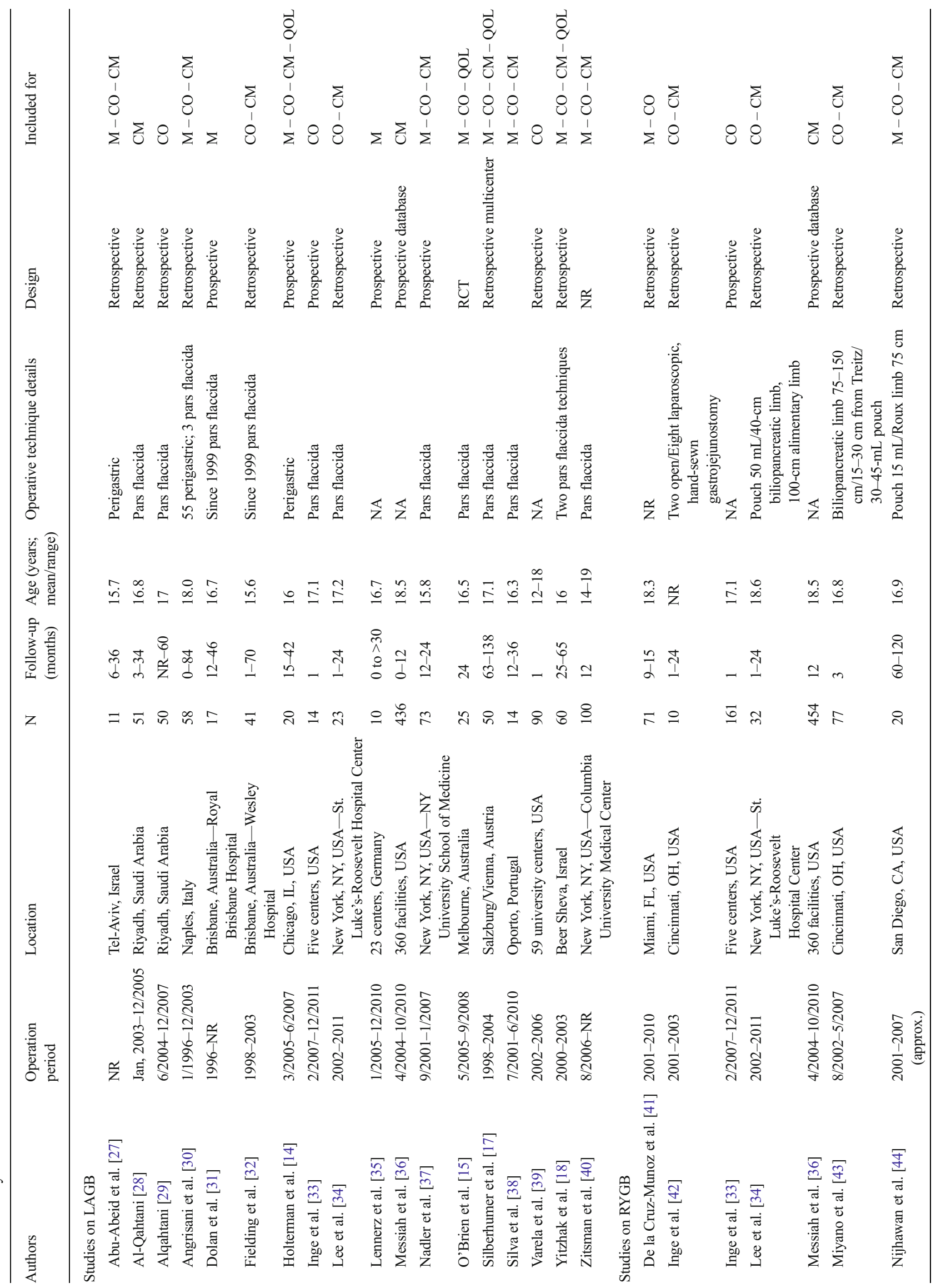




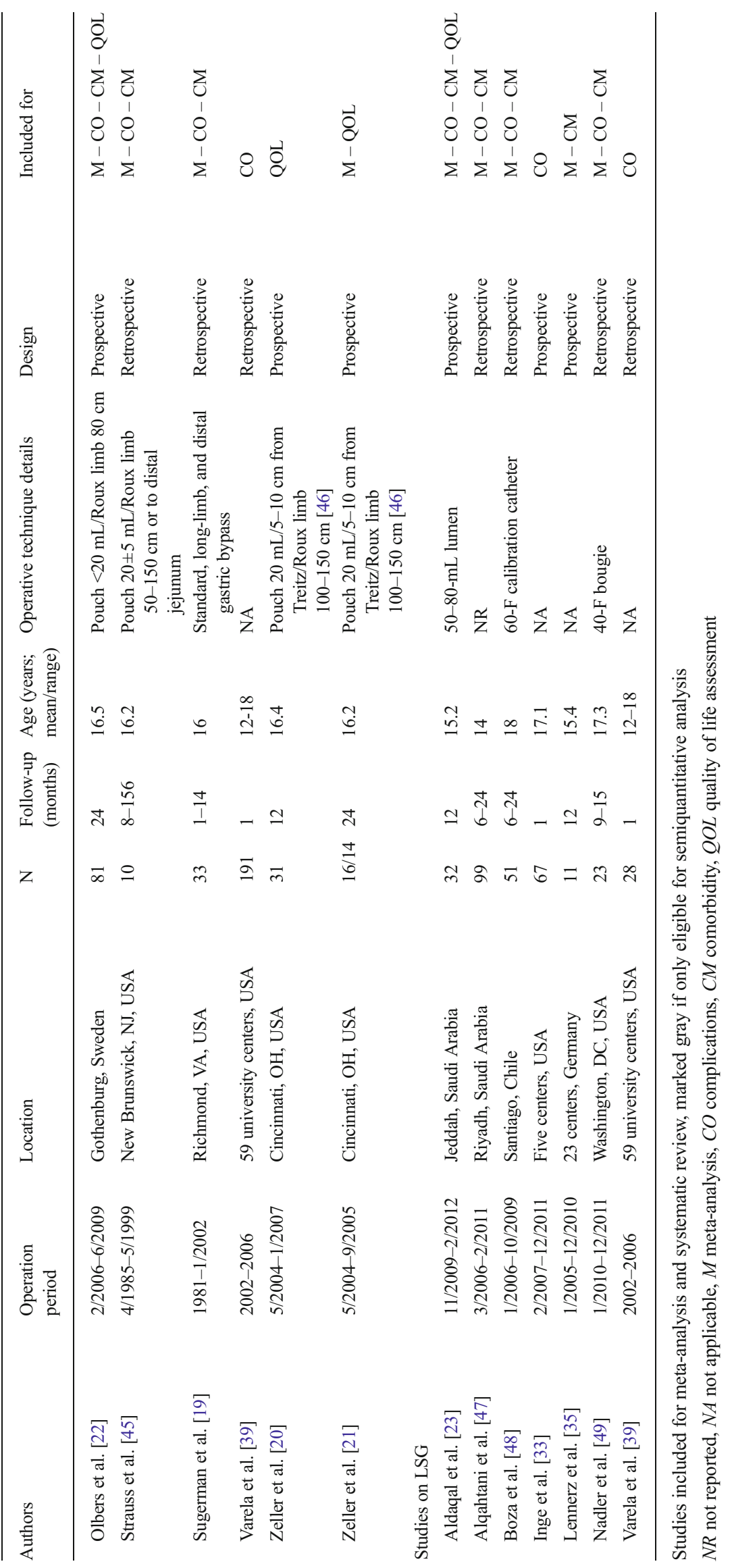


Fig. 2 Search diagram: paper retrieval schematic

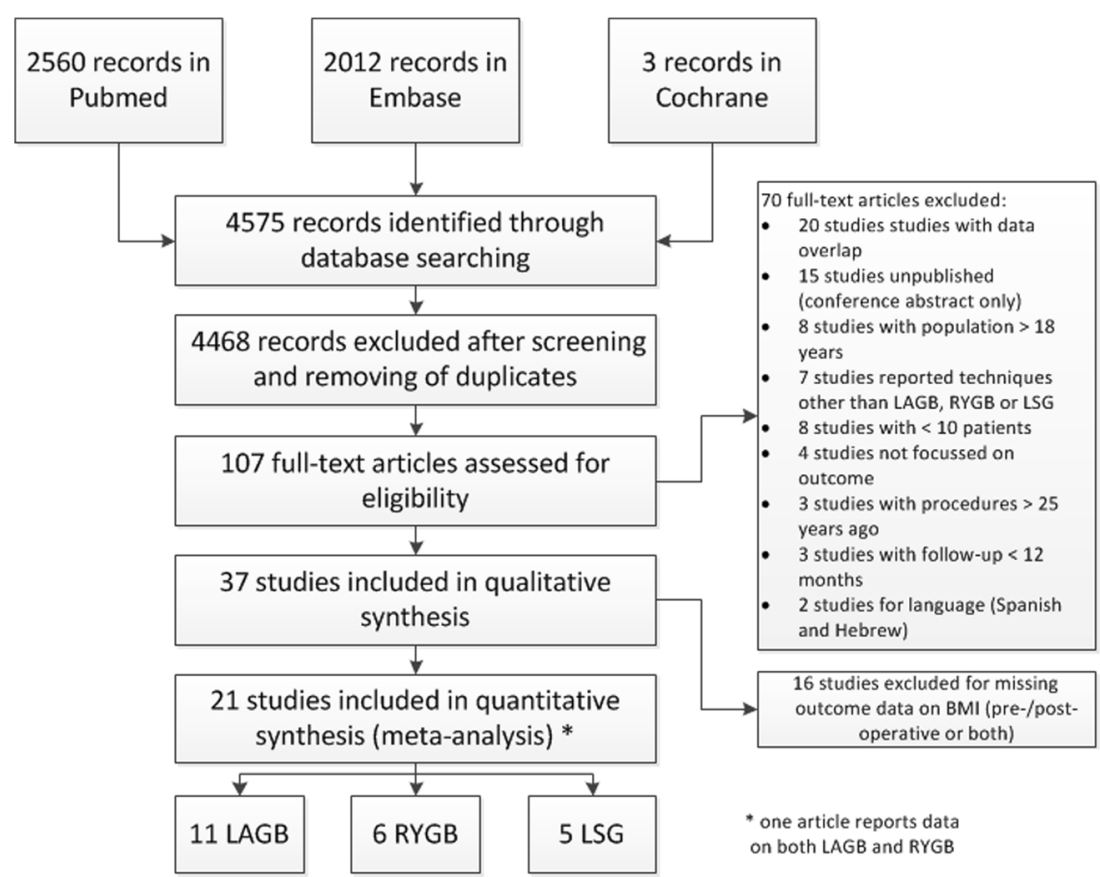

Synthesis of Results

Per procedure, a short summary is provided of weight loss, complications, comorbidity reduction, and quality of life assessment (QOL). An overview is provided in Tables 3, 4, and 5 and in Fig. 3.

\section{Laparoscopic Adjustable Gastric Band}

Weight Loss Summary BMI measure at baseline was $45.8 \mathrm{~kg} /$ $\mathrm{m}^{2}$ (44.0-47.7). The summary effect measure of BMI loss in nine studies was $11.6 \mathrm{~kg} / \mathrm{m}^{2}$ (9.8-13.4) (Fig. 3). After the first 12 months, there was no association between length of followup and excess BMI loss $(\beta=0.06, p=0.51)$. Clustering datasets by banding technique showed no differences in BMI loss (pars flaccida vs. perigastric, 11.0 vs. $10.1 \mathrm{~kg} / \mathrm{m}^{2}, p=0.61$ ).

Complications Thirteen studies report unique data on complications after gastric banding in a total of 538 patients (Table 4). No deaths occurred in any of the studies. Perioperative complications including intra-abdominal bleeding and conversion to laparotomy were reported in $0.8 \%$ and surgical site infection in $1.4 \%$. Late complications including bowel obstruction and abdominal wall hernia were reported in $1.1 \%$ of cases. During the total follow-up period (0 to 138 months), $10.5 \%$ of subjects experienced band-related complications (55/524) and $9.9 \%(17 / 172)$ gastrointestinal complaints (nausea, vomiting, GERD, diarrhea, and gallstones). There were 77 reinterventions $(14.7 \%$ ), including 3 cholecystectomies. The majority were band-related procedures like replacement or repositioning $(n=28)$, removal $(n=12)$, and port-revision $(n=16)$. Vitamin deficiencies were reported in 5 of 18 studies; oral supplements for iron, vitamin $\mathrm{D}$, folic acid, and zinc deficiencies were prescribed in 0.5 to $36 \%$ of patients, but criteria for deficiencies were poorly defined. Only 2 of 18 studies report standard postoperative vitamin supplementation, while 13 do not mention a standard policy.

Resolution of Comorbidities Out of the 18 LAGB studies included in this review, 11 report data on comorbidity resolution (Table 5). The definitions and cutoff values for comorbidities were specified in 5 of 11 studies and varied between studies. Resolution rates for hypertension, reported in nine studies, range from 22.9 to $100 \%$; six studies showed complete resolution in all patients. Nine studies report prevalence of dyslipidemia in 8 to $86 \%$, with eight reporting resolution in 0 to $100 \%$ (median $50 \%$ ) of all cases. Six out of seven studies that report on diabetes prevalence in 0 to $33 \%$, all showed $100 \%$ resolution after surgery. Resolution of prediabetes (three studies, prevalence $24-93 \%$ ) ranged from 72 to $100 \%$.

Quality of Life Holterman et al. [14] showed that $75 \%$ of the children had abnormal scores on the Pediatric Quality of Life Inventory (Peds-QL) at baseline, which improved at 12 and 18 months after surgery. The RCT by O'Brien et al. [15] showed improvements in reported physical functioning, general health, self-esteem, family activities, and change in health with the Child Health Questionnaire (CHQ CF-50) after gastric banding, while the lifestyle group improved only in general health perception. Silberhumer et al. $[16,17]$ found significant improvement after 35 months by using the BAROS and Moorehead-Ardelt Quality of Life questionnaires (both 


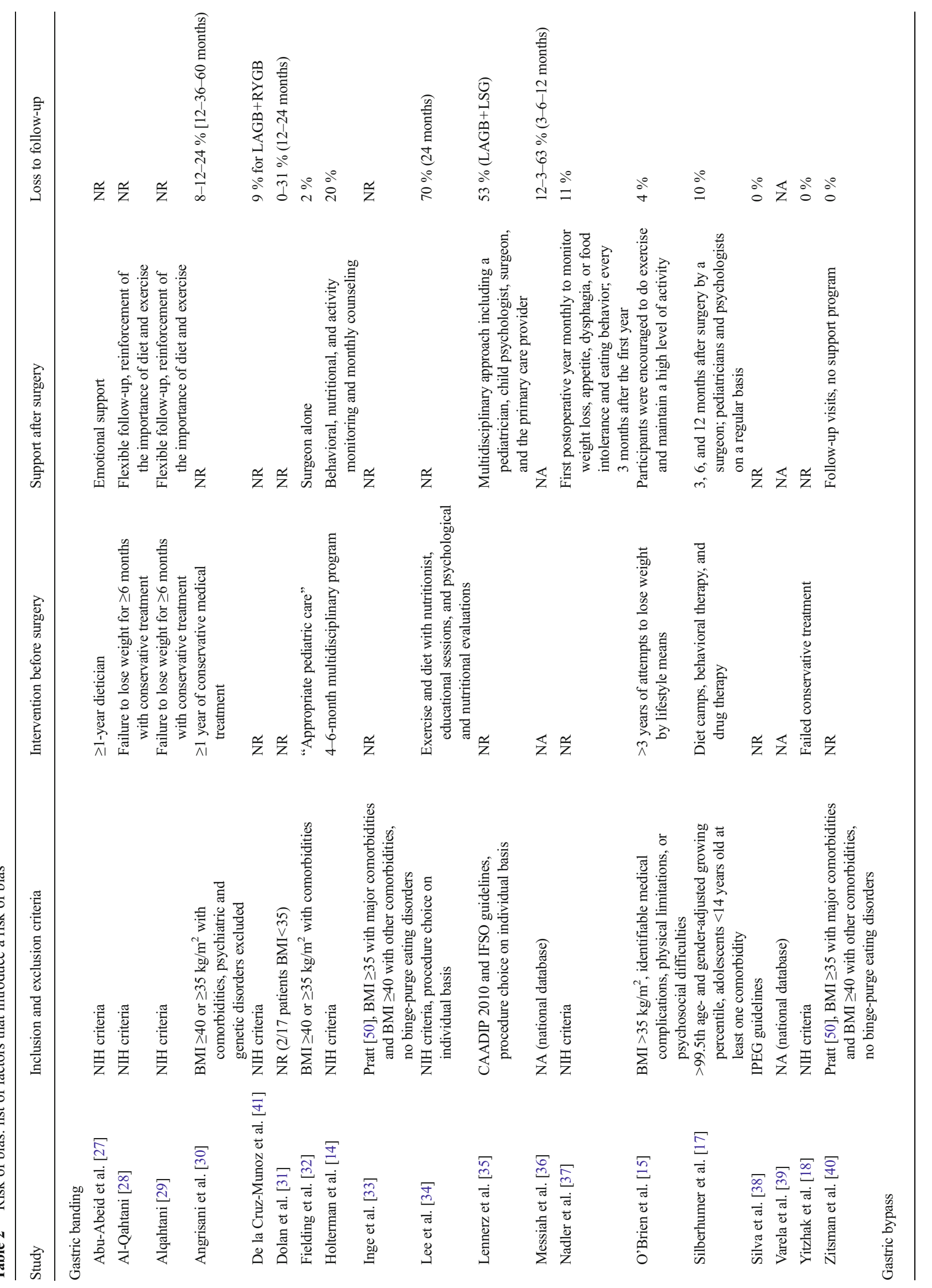




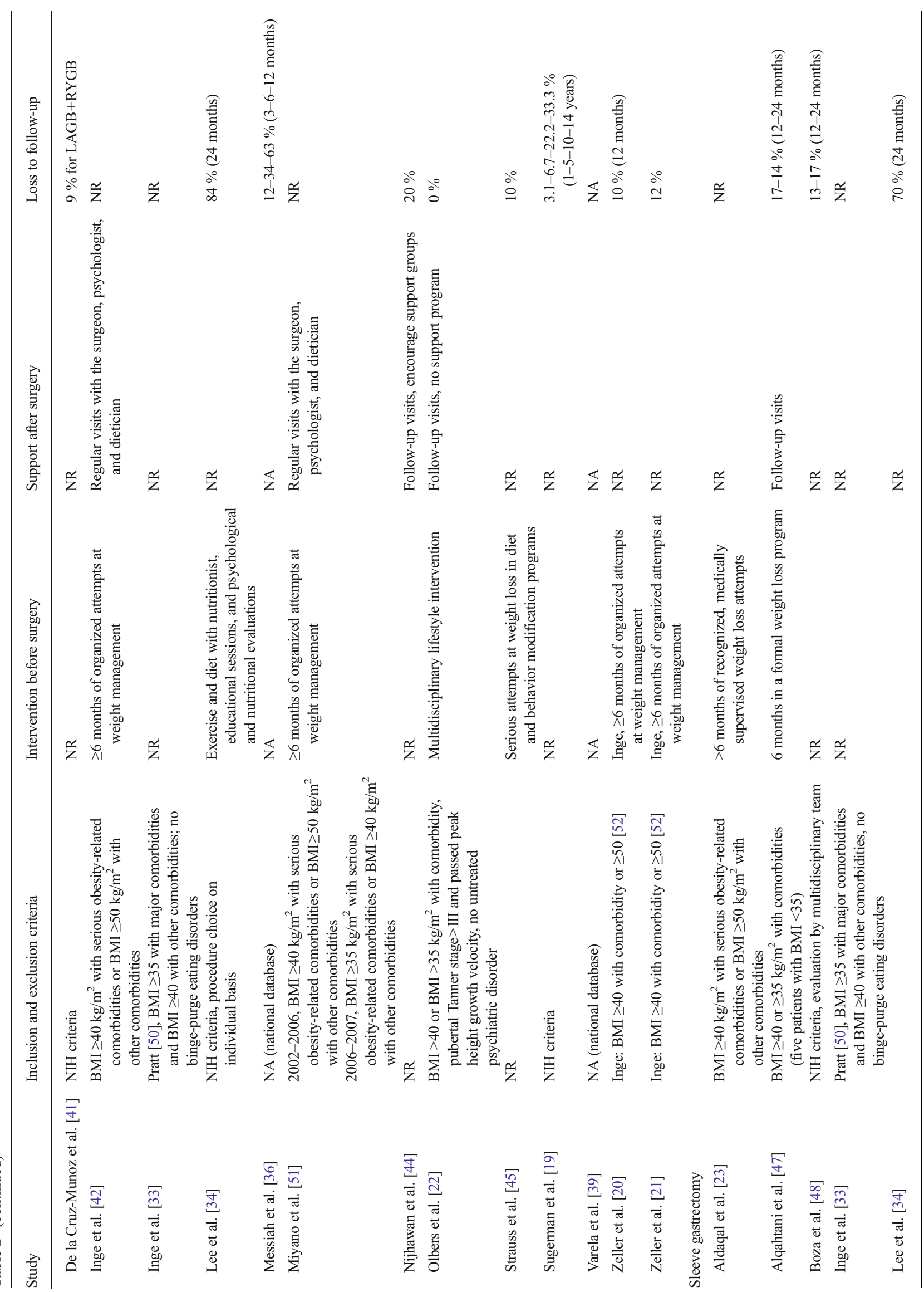


tests are not specifically validated in children) but no further changes between 3 and 5 years after surgery. Yitzhak et al. [18] report $93 \%$ improvement in physical activity and $72 \%$ improvement in social- and self-esteem with non-validated questionnaires.

Pars Flaccida Versus Perigastric Technique The LAGBrelated problems including slippage, pouch dilation, and migration - after a follow-up period of 0-7 years - do not appear to occur more in patients who were operated before the surgeons updated their techniques to the currently used pars flaccida technique $(11.2 \%(10 / 89)$ vs. $10.3 \%(45 / 435))$.

\section{Roux-en-Y Gastric Bypass}

Weight Loss The studies reporting on laparoscopic Roux-en$\mathrm{Y}$ gastric bypass have a summary BMI loss of $16.6 \mathrm{~kg} / \mathrm{m}^{2}$ (13.4-19.8) after 12 to 86 months (Table 3, Fig. 2). A follow-up period exceeding 12 months was not correlated to BMI loss $(\beta=0.04, p=0.51)$. BMI loss after RYGB was significantly higher than that after LAGB $(p=0.008)$. Mean preoperative BMI was $49.6 \mathrm{~kg} / \mathrm{m}^{2}$ (46.4-52.7) and did not differ from LAGB $(p=0.11)$.

Complications Nine studies present summarizable complication rates in a total of 495 patients. Two sudden deaths were reported in one study, 2 and 6 years after surgery, respectively, which were probably unrelated to the procedure. However, no autopsies were performed to determine the cause of death [19]. Perioperative complications including anastomotic leakage, bleeding, and conversion occurred in $5.1 \%$ and infection of the surgical site in $6.2 \%$ of patients. Late complications including obstruction, internal herniation, ulcers, and abdominal wall hernia occurred in $20.2 \%$ of patients.

Gastrointestinal complaints like nausea, vomiting, dumping, and GERD were reported in $9.3 \%$; nine patients in five studies (5.6\%) suffered from nutritional deficiencies or dehydration requiring hospitalization. Less severe vitamin deficiencies were reported in 6 of 13 studies; oral supplements for iron, vitamin A, vitamin B1, vitamin $\mathrm{B} 12$, vitamin $\mathrm{D}$, folic acid, and zinc deficiencies were used in an estimated $4-56 \%$ of patients, but criteria for deficiencies and exact numbers were poorly described. In 5 of 13 studies, postoperative vitamin supplementation was standard policy, while in seven no details are provided. The highest percentage of deficiencies occurred in the study in which no supplements were supplied.

Fifty-seven reinterventions (17.1\%) were performed including cholecystectomy in seven, endoscopic procedures (mainly balloon dilation for stricture of the anastomosis) in 18, surgery for gastrointestinal obstruction in 13, and for leak or fistula repair in six. 
Table 3 BMI loss data used for meta-analysis

\begin{tabular}{|c|c|c|c|c|c|c|}
\hline Study & $\mathrm{N}($ at FU) & FU (months) & BMI baseline & $\mathrm{SD}$ & BMI loss & $\mathrm{SD}$ \\
\hline \multicolumn{7}{|l|}{ Gastric banding } \\
\hline \multicolumn{7}{|l|}{ Perigastric technique } \\
\hline Abu-Abeid [27] & 11 & 23 & 46.4 & NR & $14.3^{\mathrm{a}}$ & NR \\
\hline Angrisani [30] & 37 & 36 & 46.1 & 6.31 & $9.1^{\mathrm{b}}$ & 4.2 \\
\hline Dolan [31] & 9 & 24 & 42.6 & 6.7 & $12.3^{\mathrm{a}}$ & 5.2 \\
\hline Holterman [14] & 12 & 18 & 50 & 10 & $9.4^{\mathrm{a}}$ & 5.4 \\
\hline \multicolumn{7}{|l|}{ Pars flaccida technique } \\
\hline Lennerz [35] & 10 & 12 & 48.1 & 9.8 & $10.1^{\mathrm{a}}$ & 9.1 \\
\hline Nadler [37] & 47 & 12 & 47.6 & 7 & $15.2^{\mathrm{b}}$ & 9.7 \\
\hline O’Brien [15] & 24 & 24 & 42.3 & 6.1 & $12.7^{\mathrm{a}}$ & NR \\
\hline Silberhumer [17] & 48 & 36 & 45.2 & 7.6 & $12.7^{\mathrm{b}}$ & 5.4 \\
\hline Silva [38] & 12 & 36 & 46.1 & 11.8 & $12.8^{\mathrm{b}}$ & 5.2 \\
\hline Yitzhak [18] & 60 & 39.5 & 43 & NR & $13^{\mathrm{a}}$ & NR \\
\hline Zitsman [40] & 47 & 12 & $50(\mathrm{M}) 48.1(\mathrm{~F})$ & NR & $6.7^{\mathrm{a}}$ & NR \\
\hline \multicolumn{7}{|l|}{ Gastric bypass } \\
\hline De la Cruz-Munoz [41] & 71 & $9-15$ & 46.2 & 5.1 & $11.3^{\mathrm{b}}$ & 5.7 \\
\hline Nijhawan [44] & 20 & 85.8 & 45.7 & NR & $17.1^{\mathrm{a}}$ & NR \\
\hline Olbers [22] & 81 & 24 & 45.5 & 6.0 & $15.3^{\mathrm{b}}$ & 6.0 \\
\hline Strauss [45] & 10 & 68.8 & 52.4 & 10.1 & $16.2^{\mathrm{c}}$ & 10.3 \\
\hline Sugerman [19] & 20 & 60 & 52 & 11 & $19^{\mathrm{a}}$ & NR \\
\hline Zeller [20] & 14 & 24 & 59.9 & 8.7 & $21.1^{\mathrm{b}}$ & 5.1 \\
\hline \multicolumn{7}{|l|}{ Sleeve gastrectomy } \\
\hline Aldaqal [23] & 32 & 12 & 49.6 & 4.9 & 20.3 & NR \\
\hline Alqahtani [47] & 76 & 6 & 49.6 (median) & 11.5 (IQR) & $14.3^{\mathrm{b}}$ & 5.5 \\
\hline Boza [48] & 34 & 24 & 38.5 & 3.7 & $12.2^{\mathrm{a}}$ & NR \\
\hline Lennerz [35] & 11 & 12 & 51.8 & 8.3 & $13.1^{\mathrm{a}}$ & 8.2 \\
\hline Nadler [57] & 13 & 6 & 52 & 9 & $10.5^{\mathrm{b}}$ & 3.8 \\
\hline
\end{tabular}

Male (M), female (F)

${ }^{\text {a }}$ From manuscript

${ }^{\mathrm{b}}$ From author

${ }^{\mathrm{c}}$ Calculated from individual data

Resolution of Comorbidities Eight of the 13 studies on RYGB report data on comorbidity resolution and/or improvement (Table 5). The definitions and cutoff values for comorbidities were specified in five of eight studies and varied between studies. The studies reporting on hypertension $(n=4)$ show 61 to $100 \%$ improvement or resolution. Six to $62 \%$ of the subjects had dyslipidemia, resolving in 56 to $100 \%$. Diabetes resolved in 79 to $100 \%$, with resolution in all subjects in five out of six studies.

Quality of Life Quality of life, reported in two studies, showed significant improvement in seven of the eight health domains on the Short Form-36 Health Survey (SF-36) at 1-year followup and significantly increased quality of life scores after 6 months, but not after 12 (assessed with the Peds-QL and
IWQOL-Kids). Depression scores were significantly less, 6 and 12 months after surgery, than before surgery [20-22].

\section{Laparoscopic Sleeve Gastrectomy}

Weight Loss Five studies present the results of the relatively new LSG technique with a follow-up between 6 and 24 months. BMI before surgery was $48.1 \mathrm{~kg} / \mathrm{m}^{2}(41.8-54.5)$, which does not differ from LAGB or RYGB patients ( $p=0.42$ and $p=0.50$, respectively). BMI loss in these studies is $14.1 \mathrm{~kg} / \mathrm{m}^{2}(10.8-17.5)$ and does not differ from LAGB and RYGB ( $p=0.17$ and $p=0.24$, respectively).

Complications Five studies including 272 patients reported two perioperative complications $(0.7 \%)$ and no mortality. 


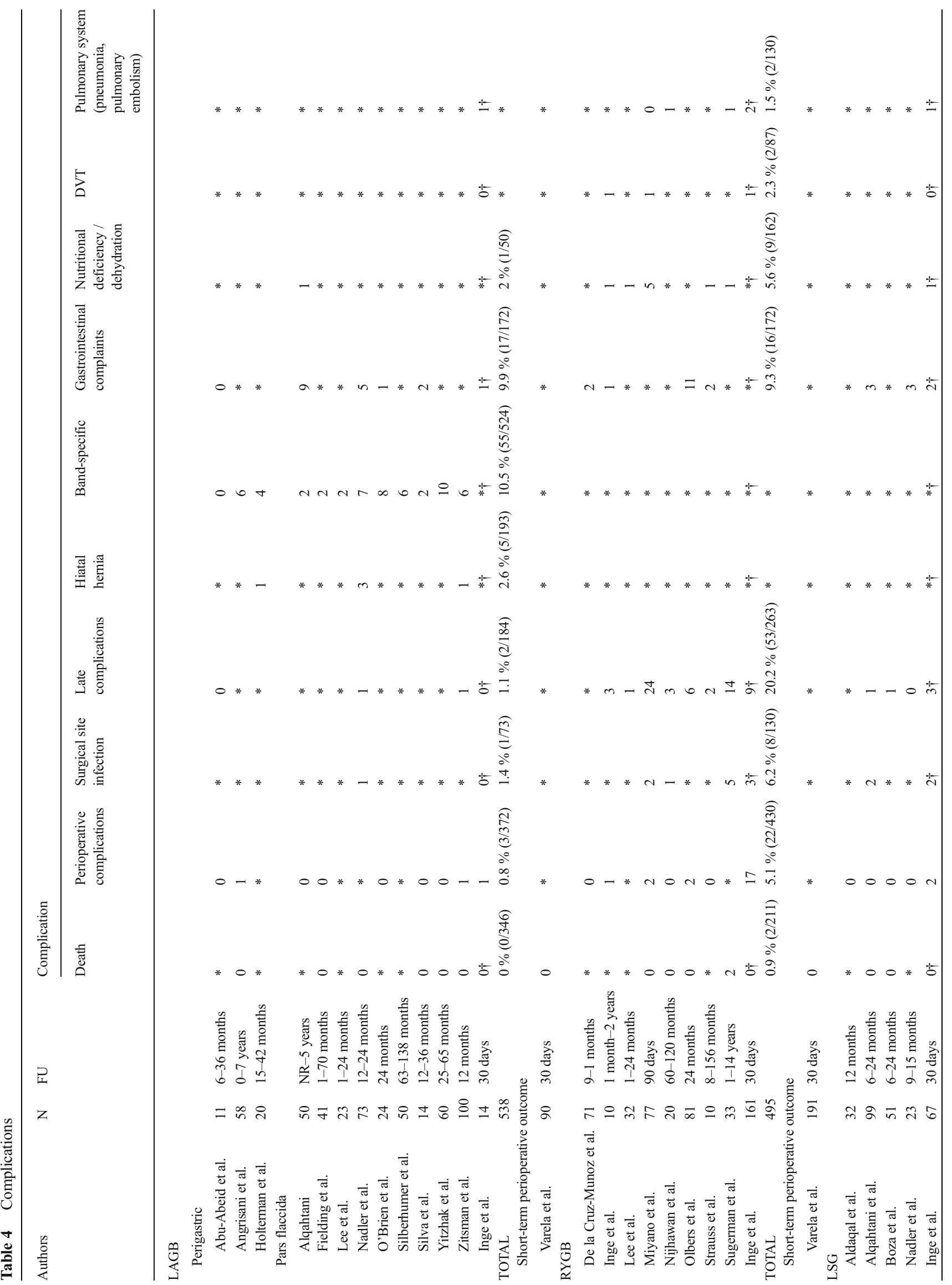




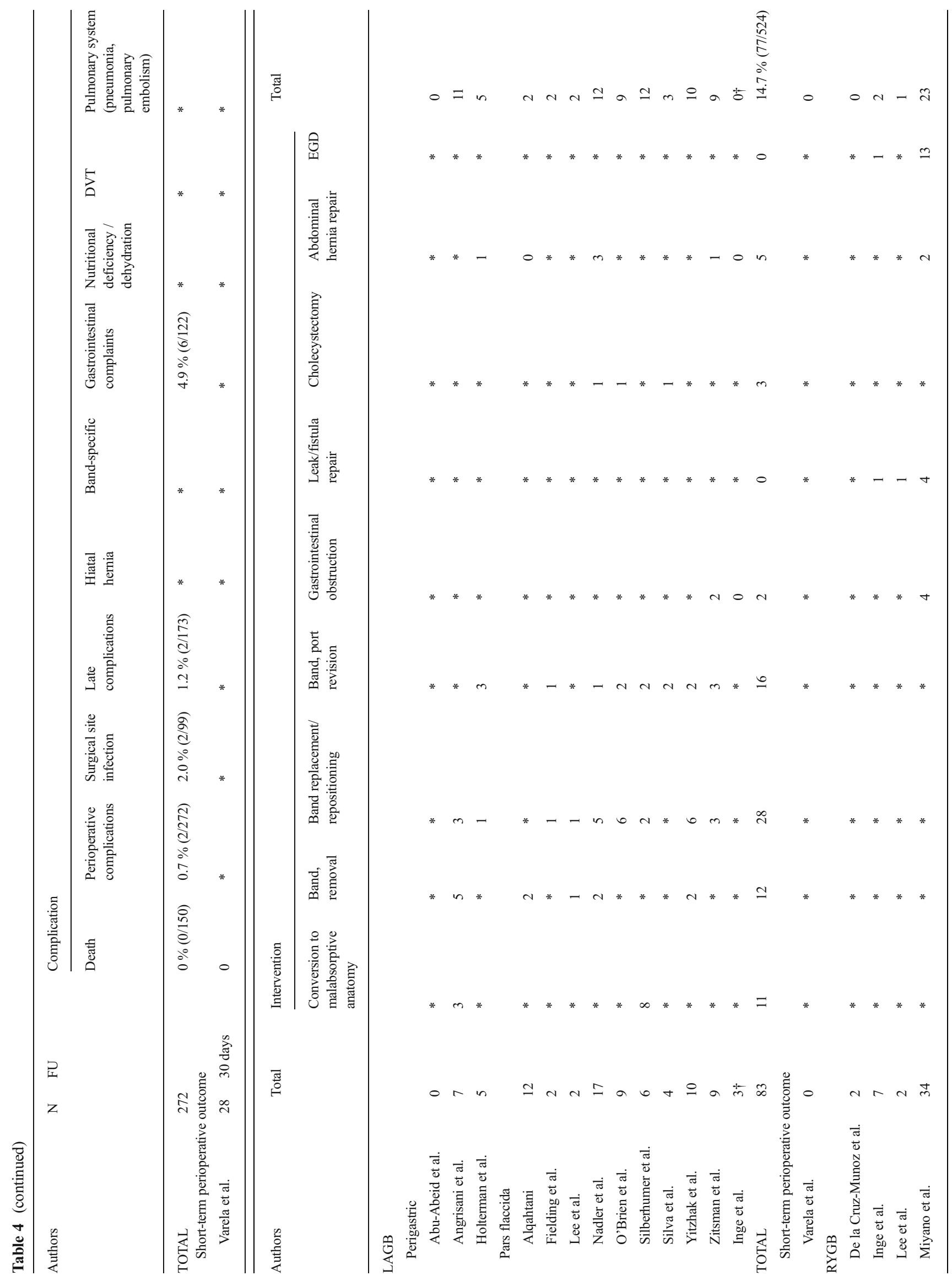




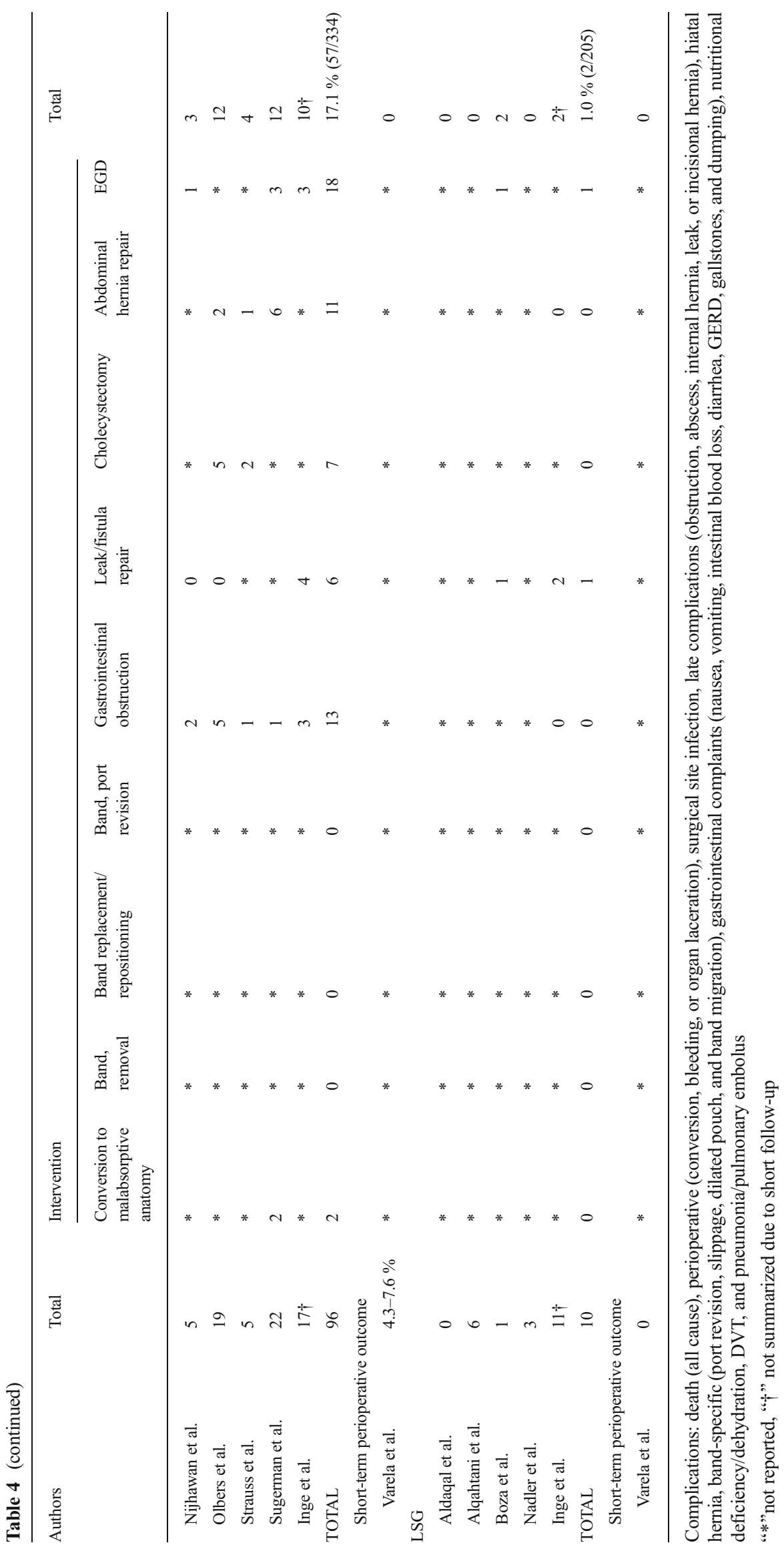




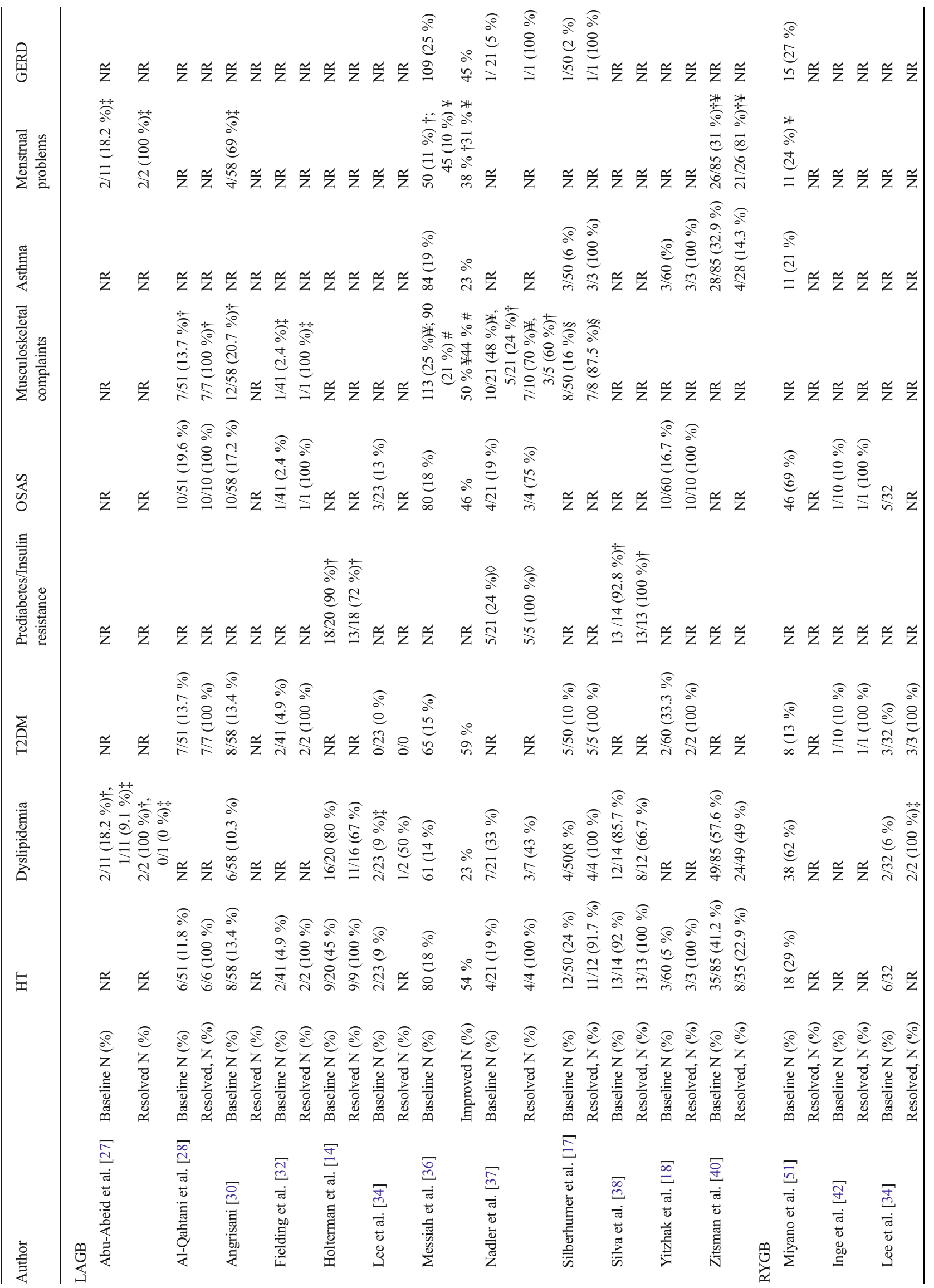




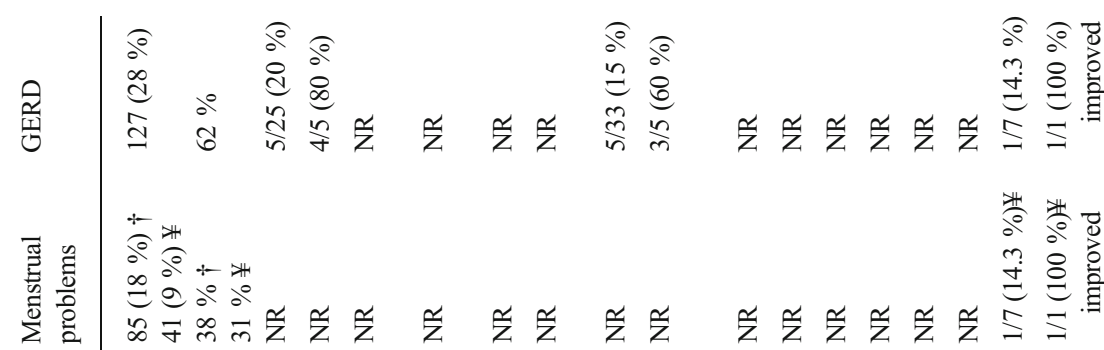

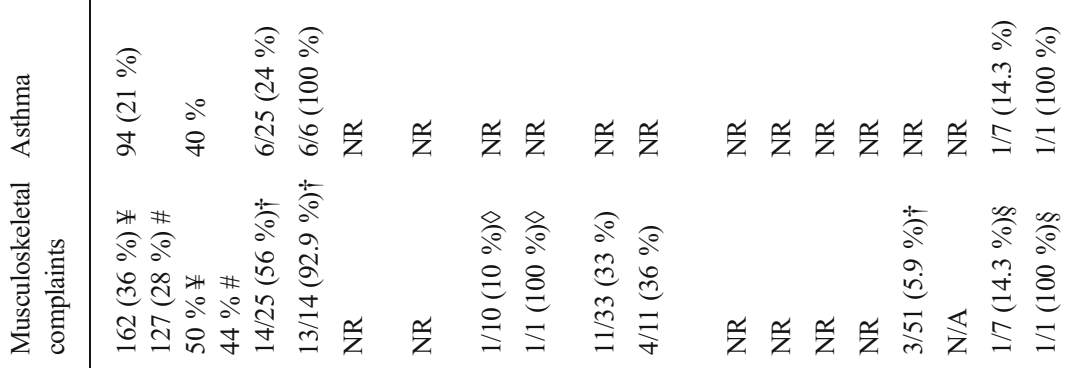

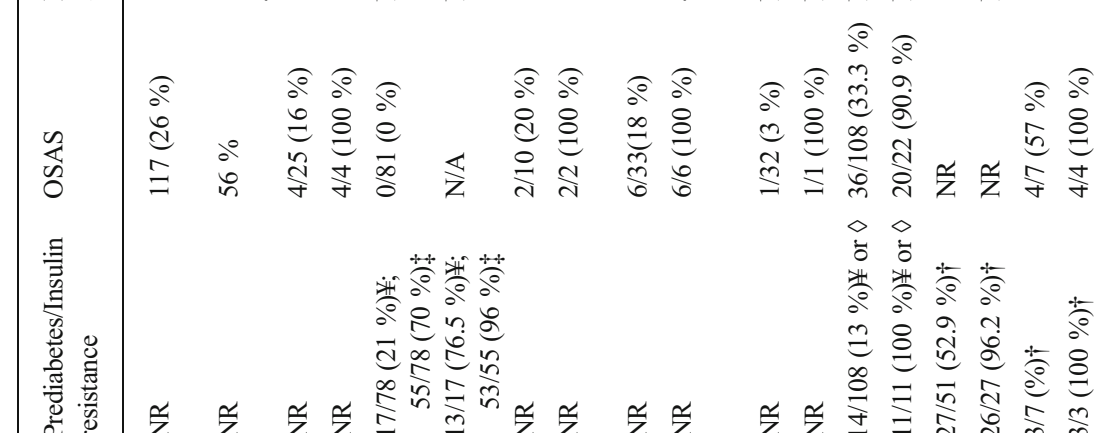

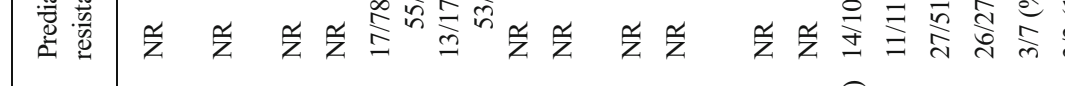

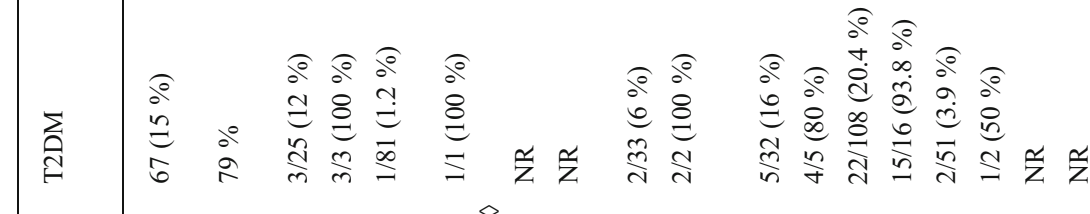

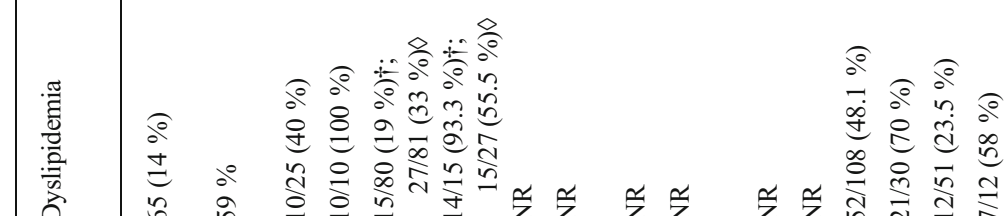

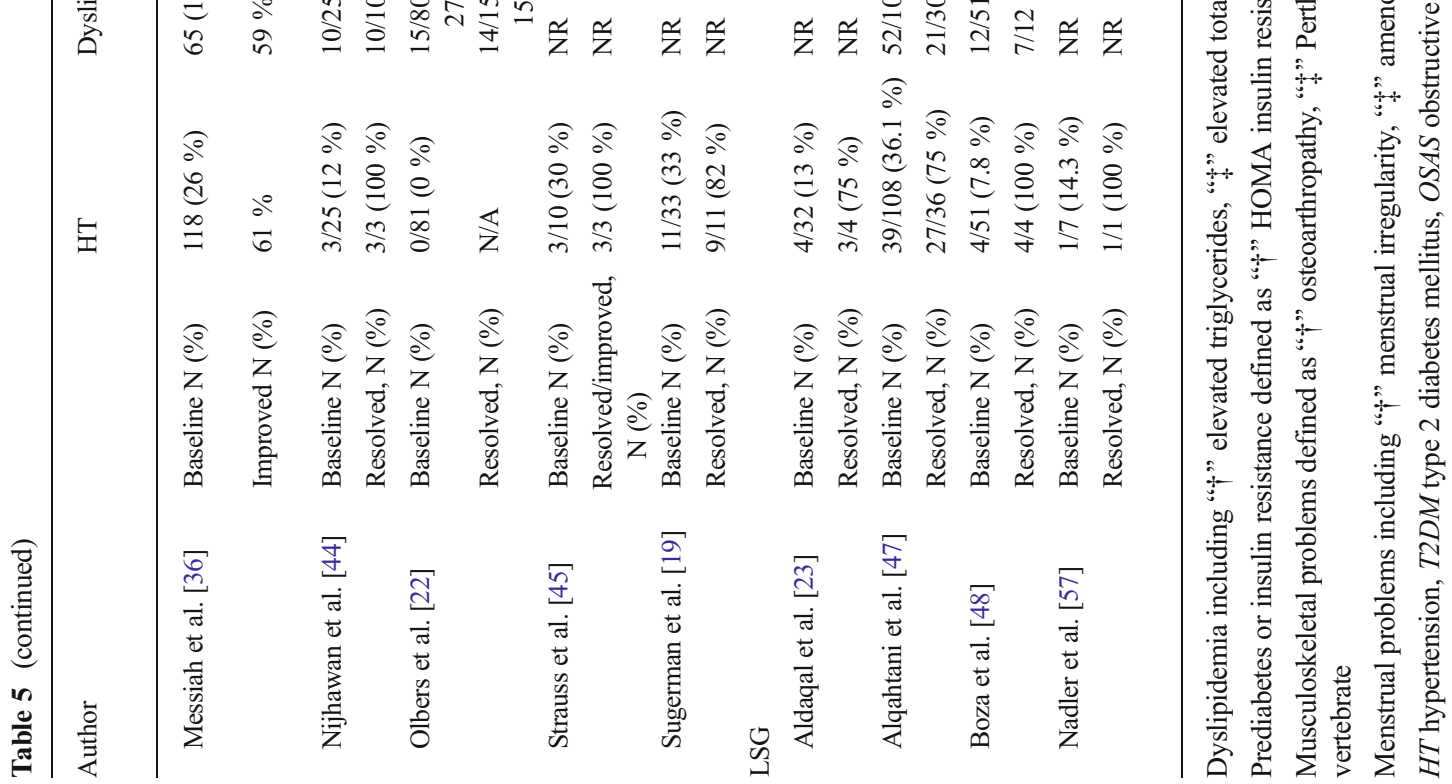


The incidence of wound infection was $2.0 \%$, and late complications occurred in $1.2 \%$, gastrointestinal complaints in $4.9 \%$ (Table 4). Postoperative vitamin supplementation was described in one of seven studies; none of the studies report whether deficiencies occurred.

Resolution of Comorbidities In four out of five studies on LSG, comorbidities are reported (Table 5). The definitions

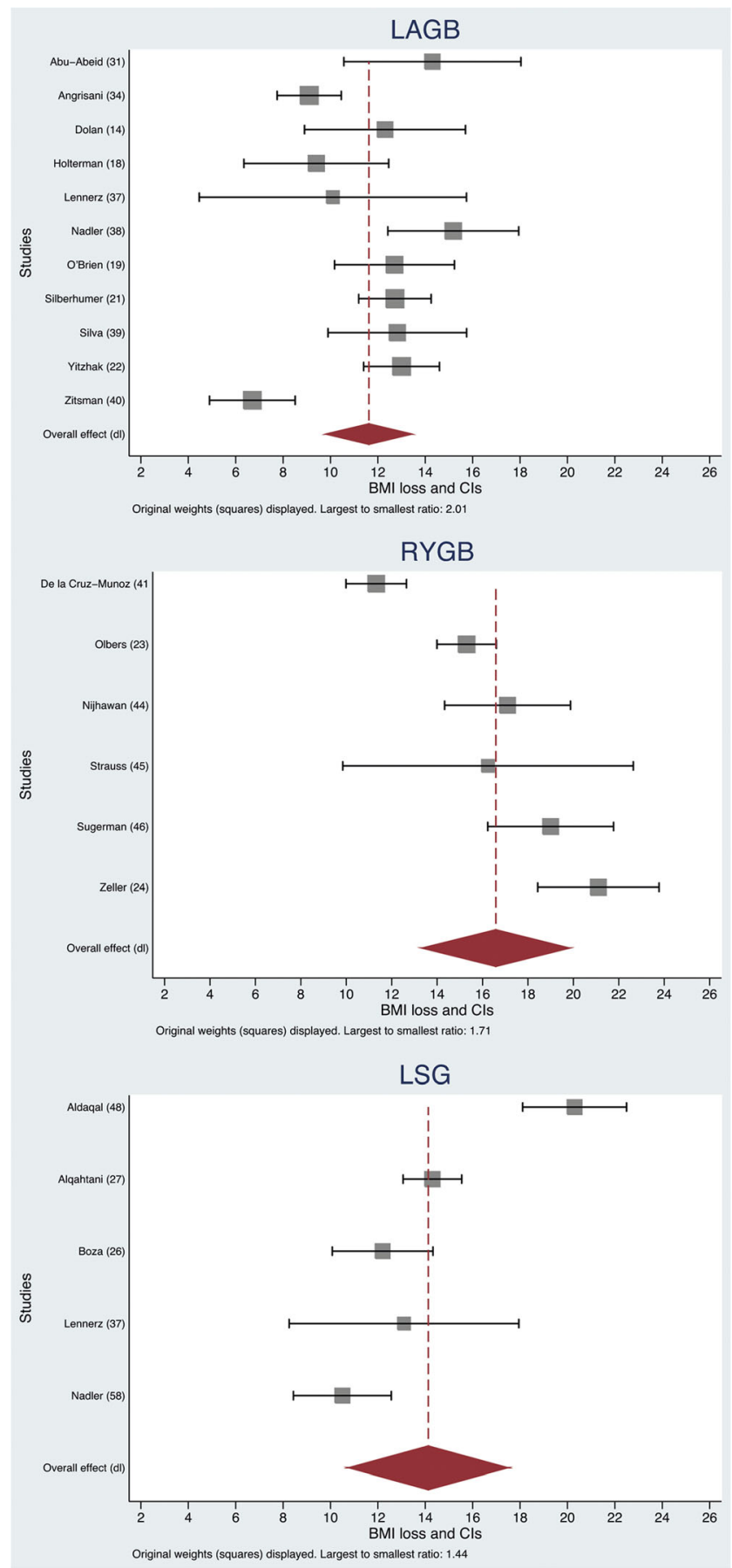

Fig. 3 Meta-analysis: forest plot for BMI loss with $95 \%$ confidence intervals and summarized means after LAGB, RYGB, and LSG and cutoff values for comorbidities were specified in two of four studies and varied between studies. Hypertension resolved in $75-100 \%$. Dyslipidemia improved, with resolution rates of 58 to $70 \%$, and diabetes, reported in three studies, resolved in 50 to $93.8 \%$.

\section{LAGB}

Funnel plot with $95 \%$ pseudo confidence limits

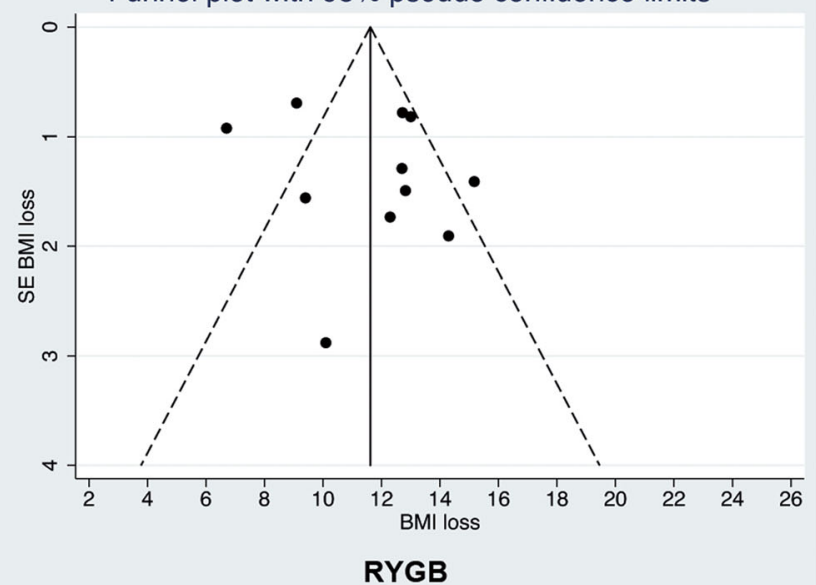

Funnel plot with 95\% pseudo confidence limits

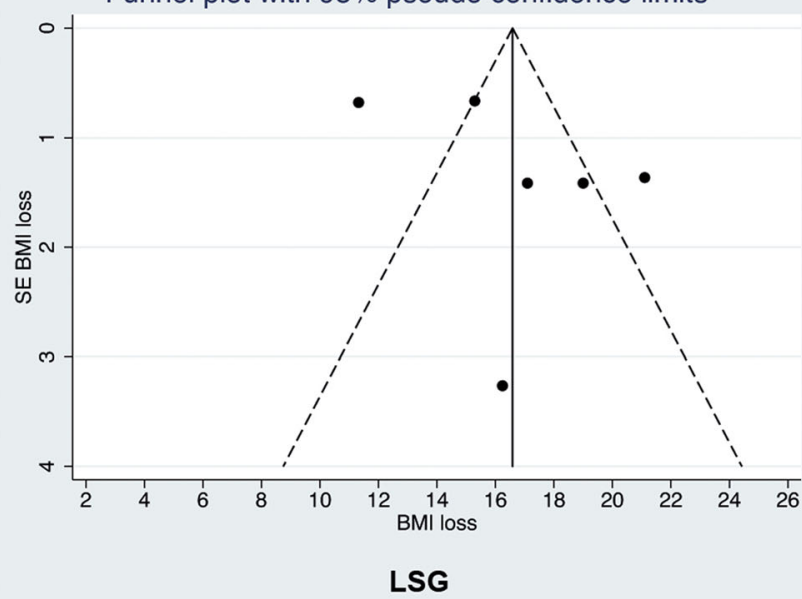

Funnel plot with $95 \%$ pseudo confidence limits

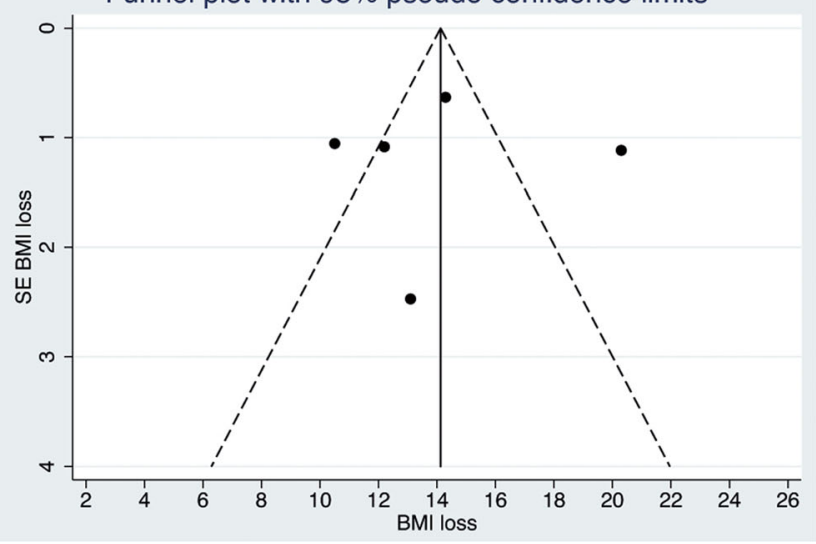

Fig. 4 Funnel plots: funnel plots of SEM of BMI loss versus BMI loss for the assessment of heterogeneity in outcome reporting. Dots outside the $95 \%$ pseudo confidence limits are indicative of heterogeneity 
Quality of Life Aldaqal et al. [23] assessed self-esteem and quality of life at baseline and 1 year after LSG with the Rosenberg self-esteem scale (RSE) and the Peds-QL. Patients improved significantly on the RSE and all six scores of the Peds-QL (including the summary score) 1 year after the procedure.

\section{Risk of Bias Across Studies}

Figure 4 shows the funnel plots for standard error of BMI loss against BMI loss in each procedure. Eight of the studies reporting on LAGB outcome are within the expected range, while one study shows more and two show less than expected BMI loss. Four RYGB studies are in the expected range, while two are not (one more and one less), and three LSG studies are in the expected range, while two are not (one more and one less).

\section{Discussion}

\section{Summary of Evidence}

The 37 studies that were eligible for systematic reviewing represent the increasing interest in bariatric surgery in morbidly obese adolescents, although the studies were mainly observational and varied in quality. To ensure that the meta-analysis was based on valid data and solidly compares surgical methods, we reported only peer-reviewed published studies and obtained additional data from the authors of nine studies.

All three procedures lead to significant weight loss in morbidly obese adolescents, and similar to a large Swedish study in adults, weight loss is most pronounced after RYGB [9]. This seems to persist after both RYGB and LAGB. For LSG studies, long-term follow-up is not yet available. While adverse events are relatively mild and long-term complication rates are acceptable, they are more frequent and more serious after RYGB than after LAGB. In the currently available follow-up after LSG, the rate of adverse events appears to be similar to that after LAGB. Although a healthy nutritional status in adolescents is important to prevent developmental and growth deficiencies, standard postoperative vitamin supplementation regimens and the occurrence of deficiencies are not reported in most studies (not at all in LSG studies). However, more and more severe deficiencies occur after RYGB than after LAGB.

Reduction of comorbidity, which is pivotal for health gain, is impressive in all techniques, and QOL consistently showed improvement, although follow-up up to 24 months may not be enough to capture negative long-term effects in life after bariatric surgery. The difference in adults between adverse events of the old perigastric LAGB technique and the more recently adapted pars flaccida technique [24] is not reproduced reviewing young patients.

\section{Limitations}

Funnel plots show heterogeneity of the data but no indication of publication bias due to underreporting of poor outcomes. A limitation of the currently available literature is the lack of high-quality, prospective randomized controlled trials, which increases the risk of bias and therefore introduces heterogeneity. Assessment of the three fundamental domains in risk of bias in observational studies (appropriate selection of participants, appropriate measurement of variables, and appropriate control of confounding) shows that studies are heterogeneous in patient selection, in preoperative and postoperative treatment protocol and that loss-to follow-up is substantial. Furthermore, reduction of comorbidity receives sufficient attention in most studies, but varying and lacking definitions of comorbidity introduce another possible source of bias. The similarity in outcome in all studies, however, strengthens our conclusion that the current methods of summarizing BMI loss, complication rate, and reduction of comorbidity are indicative of the true outcome.

\section{Conclusions}

This review is the first that has retrieved sufficient data for meta-analysis of BMI loss by contacting all authors of included studies, to enable a solid statistical analysis. All three analyzed bariatric surgical techniques - laparoscopic adjustable gastric banding, Roux-en-Y gastric bypass, and laparoscopic sleeve gastrectomy - result in substantial weight loss and improvement of comorbidity in the short to medium term. This indicates that, considering the acceptable complication rate, surgical intervention is applicable in appropriately selected adolescents. While BMI loss after RYGB is superior, a higher rate of adverse events and reinterventions has to be taken into account. We recognize that RYGB is currently considered in the treatment of adolescents with a more extreme BMI $\left(>50 \mathrm{~kg} / \mathrm{m}^{2}\right)$, while LAGB and LSG are applied when obesity is less extreme.

The quality of the available literature is limited. In the current climate where availability of bariatric surgery for morbidly obese children is already increasing, randomized controlled trials comparing bariatric surgery with standard conservative treatment are difficult to perform. Currently, seven active studies are registered in ClinicalTrials.gov assessing the effects of bariatric surgery in adolescents, including one randomized controlled trial. We recommend the involved researchers to use solid outcome reporting strategies and strongly support the pleas for standardized weight loss reporting $[25,26]$. 
Acknowledgments Many authors were willing to correspond about their findings and provided additional data. We would like to thank A.R. Alqahtani, L. Angrisani, N. de la Cruz-Muñoz, M. Elahmedi, E. Gronowitz, M. Lorenzo, S.E. Messiah, E.P. Nadler, G. Prager, G.R. Silberhumer, G.M. Silva, H.A. Youn, M.H. Zeller, and J.L. Zitsman for their cooperation. Their input makes this meta-analysis unique.

Conflict of Interest The authors declare that they have no conflict of interest.

\section{Statement of Informed Consent Does not apply.}

Statement of Human and Animal Rights For this article, no studies with human participants or animals were performed by any of the authors.

Funding There was no funding for this systematic review.

Open Access This article is distributed under the terms of the Creative Commons Attribution License which permits any use, distribution, and reproduction in any medium, provided the original author(s) and the source are credited.

\section{References}

1. Obesity: preventing and managing the global epidemic. Report of a WHO consultation. World Health Organ Tech Rep Ser. 2000;894:ixii, 1-253. PubMed PMID: 11234459.

2. Ogden CL, Flegal KM, Carroll MD, et al. Prevalence and trends in overweight among US children and adolescents, 1999-2000. JAMA. 2002;288(14):1728-32. PubMed PMID: 12365956.

3. Ogden CL, Carroll MD, Kit BK, et al. Prevalence of obesity and trends in body mass index among US children and adolescents, 1999-2010. JAMA. 2012;307(5):483-90. PubMed PMID: 22253364.

4. Rocchini AP. Childhood obesity and coronary heart disease. N Engl J Med. 2011;365(20):1927-9. PubMed PMID: 22087684.

5. Juonala M, Magnussen CG, Berenson GS, et al. Childhood adiposity, adult adiposity, and cardiovascular risk factors. N Engl J Med. 2011;365(20):1876-85. PubMed PMID: 22087679.

6. Russell-Mayhew S, McVey G, Bardick A, et al. Mental health, wellness, and childhood overweight/obesity. J Obes. 2012;2012:281801. PubMed PMID: 22778915. Pubmed Central PMCID: 3388583.

7. Abdullah A, Wolfe R, Stoelwinder JU, et al. The number of years lived with obesity and the risk of all-cause and cause-specific mortality. Int J Epidemiol. 2011;40(4):985-96. PubMed PMID: 21357186.

8. Oude Luttikhuis H, Baur L, Jansen H, et al. Interventions for treating obesity in children. Cochrane Database Syst Rev (Online). 2009 (1): CD001872. PubMed PMID: 19160202.

9. Sjöström L. Review of the key results from the Swedish Obese Subjects (SOS) trial - a prospective controlled intervention study of bariatric surgery. J Intern Med. 2013;273(3):219-34.

10. International Pediatric Endosurgery G. IPEG guidelines for surgical treatment of extremely obese adolescents. J Laparoendosc Adv Surg Tech A. 2009;19 Suppl 1.

11. Treadwell JR, Sun F, Schoelles K. Systematic review and metaanalysis of bariatric surgery for pediatric obesity. Ann Surg. 2008;248(5):763-76. PubMed PMID: 18948803.

12. Moher D, Liberati A, Tetzlaff J, et al. Preferred reporting items for systematic reviews and meta-analyses: the PRISMA statement. PLoS Med. 2009;6(7):e1000097. PubMed PMID: 19621072. Pubmed Central PMCID: PMC2707599.

13. Stroup DF, Berlin JA, Morton SC, et al. Meta-analysis of observational studies in epidemiology: a proposal for reporting. Meta- analysis Of Observational Studies in Epidemiology (MOOSE) group. JAMA. 2000;283(15):2008-12. PubMed PMID: 10789670.

14. Holterman AX, Browne A, Tussing L, et al. A prospective trial for laparoscopic adjustable gastric banding in morbidly obese adolescents: an interim report of weight loss, metabolic and quality of life outcomes. J Pediatr Surg. 2010;45(1):74-8. discussion 8-9. PubMed PMID: 20105583.

15. O’Brien PE, Sawyer SM, Laurie C, et al. Laparoscopic adjustable gastric banding in severely obese adolescents: a randomized trial. JAMA. 2010;303(6):519-26. PubMed PMID: 20145228.

16. Silberhumer GR, Miller K, Kriwanek S, et al. Laparoscopic adjustable gastric banding in adolescents: the Austrian experience. Obes Surg. 2006;16(8):1062-7. PubMed PMID: 16901361.

17. Silberhumer GR, Miller K, Pump A, et al. Long-term results after laparoscopic adjustable gastric banding in adolescent patients: follow-up of the Austrian experience. Surg Endosc. 2011;25(9): 2993-9. PubMed PMID: 21573716.

18. Yitzhak A, Mizrahi S, Avinoach E. Laparoscopic gastric banding in adolescents. Obes Surg. 2006;16(10):1318-22. PubMed PMID: 17059740.

19. Sugerman HJ, Sugerman EL, DeMaria EJ, et al. Bariatric surgery for severely obese adolescents. J Gastrointest Surg. 2003;7(1):102.

20. Zeller MH, Modi AC, Noll JG, et al. Psychosocial functioning improves following adolescent bariatric surgery. Obesity (Silver Spring). 2009;17(5):985-90. PubMed PMID: 19165158.

21. Zeller MH, Reiter-Purtill J, Ratcliff MB, et al. Two-year trends in psychosocial functioning after adolescent Roux-en-Y gastric bypass. Surg Obes Relat Dis. 2011;7(6):727-32. PubMed PMID: 21497142.

22. Olbers T, Gronowitz E, Werling M, et al. Two-year outcome of laparoscopic Roux-en-Y gastric bypass in adolescents with severe obesity: results from a Swedish Nationwide Study (AMOS). Int J Obes (2005). 2012;36(11):1388-95. PubMed PMID: 23007037.

23. Aldaqal S, Sehlo M. Self-esteem and quality of life in adolescents with extreme obesity in Saudi Arabia: the effect of weight loss after laparoscopic sleeve gastrectomy. Gen Hosp Psychiatry. 2013;35(3):259-64.

24. O'Brien PE, Dixon JB, Laurie C, et al. A prospective randomized trial of placement of the laparoscopic adjustable gastric band: comparison of the perigastric and pars flaccida pathways. Obes Surg. 2005;15(6):820-6. PubMed PMID: 15978154.

25. Montero PN, Stefanidis D, Norton HJ, et al. Reported excess weight loss after bariatric surgery could vary significantly depending on calculation method: a plea for standardization. Surg Obes Relat Dis. 2011;7(4):531-4.

26. Belle SH, Berk PD, Courcoulas AP, et al. Reporting weight change: standardized reporting accounting for baseline weight. Surg Obes Relat Dis. 2013;9(5):782-9.

27. Abu-Abeid S, Gavert N, Klausner JM, et al. Bariatric surgery in adolescence. J Pediatr Surg. 2003;38(9):1379-82. PubMed PMID: 14523824.

28. Al-Qahtani AR. Laparoscopic adjustable gastric banding in adolescent: safety and efficacy. J Pediatr Surg. 2007;42(5):894-7. PubMed PMID: 17502207.

29. Alqahtani A. Robotic gastric banding in children and adolescents: a comparative study. Surg Endosc. 2011;25(11):3647-51. PubMed PMID: 21638172.

30. Angrisani L, Favretti F, Furbetta F, et al. Obese teenagers treated by Lap-Band System: the Italian experience. Surgery. 2005;138(5):87781. PubMed PMID: 16291388.

31. Dolan K, Creighton L, Hopkins G, et al. Laparoscopic gastric banding in morbidly obese adolescents. Obes Surg. 2003;13(1): 101-4. PubMed PMID: 12630622.

32. Fielding GA, Duncombe JE. Laparoscopic adjustable gastric banding in severely obese adolescents. Surg Obes Relat Dis. 2005;1(4):399405. discussion -7. PubMed PMID: 16925257.

33. Inge $\mathrm{T}$, Zeller $\mathrm{M}$, Jenkins $\mathrm{T}$, et al. Perioperative outcomes of adolescents undergoing bariatric surgery: the teen-longitudinal assessment 
of bariatric surgery (Teen-LABS) study. JAMA Pediatr. 2014;168(1): 47-53.

34. Lee D, Guend H, Park K, et al. Outcomes of laparoscopic Roux-en-Y gastric bypass versus laparoscopic adjustable gastric banding in adolescents. Obes Surg. 2012;22(12):1859-64.

35. Lennerz B, Wabitsch M, Lippert $H$, et al. Bariatric surgery in adolescents and young adults-safety and effectiveness in a cohort of 345 patients. Int J Obes (2005). 2013.

36. Messiah S, Lopez-Mitnik G, Winegar D, et al. Changes in weight and co-morbidities among adolescents undergoing bariatric surgery: 1year results from the Bariatric Outcomes Longitudinal Database. Surg Obes Relat Dis. 2013;9(4):503-13.

37. Nadler EP, Youn HA, Ren CJ, et al. An update on 73 US obese pediatric patients treated with laparoscopic adjustable gastric banding: comorbidity resolution and compliance data. J Pediatr Surg. 2008;43(1):141-6. PubMed PMID: 18206472.

38. Silva GM, Osorio A, Pereira F, et al. Effect of laparoscopic adjustable gastric banding on modifiable cardiovascular risk factors in extremely obese adolescents. Obes Surg. 2012;22(6):991-4. PubMed PMID: 22491997.

39. Varela JE, Hinojosa MW, Nguyen NT. Perioperative outcomes of bariatric surgery in adolescents compared with adults at academic medical centers. Surg Obes Relat Dis. 2007;3(5):537-40. discussion 41-2. PubMed PMID: 17903775.

40. Zitsman JL, Fennoy I, Witt MA, et al. Laparoscopic adjustable gastric banding in adolescents: short-term results. J Pediatr Surg. 2011;46(1): 157-62. PubMed PMID: 21238658.

41. De La Cruz-Munoz N, Lopez-Mitnik G, Arheart KL, et al. Effectiveness of bariatric surgery in reducing weight and body mass index among Hispanic adolescents. Obes Surg. 2012. PubMed PMID: 22918552.

42. Inge $\mathrm{TH}$, Garcia $\mathrm{V}$, Daniels $\mathrm{S}$, et al. A multidisciplinary approach to the adolescent bariatric surgical patient. J Pediatr Surg. 2004;39(3): 442-7. discussion 6-7. PubMed PMID: 15017567.

43. Zwintscher N, Azarow K, Horton J, et al. The increasing incidence of adolescent bariatric surgery. J Pediatr Surg. 2013;48(12):2401-7.

44. Nijhawan S, Martinez T, Wittgrove AC. Laparoscopic gastric bypass for the adolescent patient: long-term results. Obes Surg. 2012;22(9): 1445-9. PubMed PMID: 22638680.

45. Strauss RS, Bradley LJ, Brolin RE. Gastric bypass surgery in adolscents with morbid obesity. J Pediatr. 2001;138(4):499-504. PubMed PMID: 2001147165.
46. Cozacov Y, Roy M, Moon S, et al. Mid-term results of laparoscopic sleeve gastrectomy and Roux-en-Y gastric bypass in adolescent patients. Obes Surg. 2014.

47. Alqahtani AR, Antonisamy B, Alamri H, et al. Laparoscopic sleeve gastrectomy in 108 obese children and adolescents aged 5 to 21 years. Ann Surg. 2012;256(2):266-73. PubMed PMID: 22504281.

48. Boza C, Viscido G, Salinas J, et al. Laparoscopic sleeve gastrectomy in obese adolescents: results in 51 patients. Surg Obes Relat Dis. 2012;8(2):133-7. discussion 7-9. PubMed PMID: 22433934.

49. Nadler EP, Qureshi FG, Barefoot L. Early results after laparoscopic sleeve gastrectomy in adolescents with morbid obesity. J Surg Res. 2012 February; Conference: 7th Annual Academic Surgical Congress of the Association for Academic Surgery, AAS and the Society of University Surgeons, SUS Las Vegas, NV United States. Conference Start: 20120214 Conference End: 20120216. Conference Publication: (var.pagings). 172 (2):318-9. PubMed PMID: 70651482.

50. Pratt J, Lenders C, Dionne E, et al. Best practice updates for pediatric/ adolescent weight loss surgery. Obesity (Silver Spring, Md). 2009;17(5):901-10.

51. Miyano G, Jenkins T, Xanthakos S, et al. Perioperative outcome of laparoscopic Roux-en-Y gastric bypass: a children's hospital experience. J Pediatr Surg. 2013;48(10):2092-8.

52. Inge T, Krebs N, Garcia V, et al. Bariatric surgery for severely overweight adolescents: concerns and recommendations. Pediatrics. 2004;114(1):217-23.

53. Oberbach A, von Bergen M, Bluher S, et al. Combined serum proteomic and metabonomic profiling after laparoscopic sleeve gastrectomy in children and adolescents. J Laparoendosc Adv Surg Tech A. 2012;22(2):184-8. PubMed PMID: 21958229.

54. NIH conference. Gastrointestinal surgery for severe obesity. Consensus Development Conference Panel. Ann Inter Med. 1991;115(12):956-61.

55. CA-ADIP. S3-Leitlinie: Chirurgie der Adipositas. http://www. dgavde/fileadmin/media/texte_pdf/caadip/leitlinie-chirurgie-deradipositas_2010-06.pdf. 2010.

56. Fried M, Yumuk V, Oppert J-M, et al. Interdisciplinary European guidelines on metabolic and bariatric surgery. Obes Facts. 2013;6(5):449-68.

57. Nadler EP, Barefoot LC, Qureshi FG. Early results after laparoscopic sleeve gastrectomy in adolescents with morbid obesity. Surgery. 2012;152(2):212-7. PubMed PMID: 22828142. 